\title{
A comparison between the effort-reward imbalance and demand control models
}

\author{
Aleck S Ostry*1, Shona Kelly ${ }^{1}$, Paul A Demers ${ }^{1}$, Cameron Mustard ${ }^{2}$ and \\ Clyde Hertzman ${ }^{1}$
}

\begin{abstract}
Address: ${ }^{1}$ Department of Health Care and Epidemiology, University of British Columbia, Canada and ${ }^{2}$ Institute of Work and Health (Toronto), Canada

Email: Aleck S Ostry* - ostry@interchange.ubc.ca; Shona Kelly - skelly@interchange.ubc.ca; Paul A Demers - pdemers@interchange.ubc.ca; Cameron Mustard - cmustard@iwh.on.ca; Clyde Hertzman - hertzman@interchange.ubc.ca

* Corresponding author
\end{abstract}

Published: 27 February 2003

BMC Public Health 2003, 3:10

This article is available from: http://www.biomedcentral.com/147I-2458/3/10

(c) 2003 Ostry et al; licensee BioMed Central Ltd. This is an Open Access article: verbatim copying and redistribution of this article are permitted in all media for any purpose, provided this notice is preserved along with the article's original URL.
Received: 29 October 2002

Accepted: 27 February 2003

\begin{abstract}
Background: To compare the predictive validity of the demand/control and reward/imbalance models, alone and in combination with each other, for self-reported health status and the selfreported presence of any chronic disease condition.

Methods: Self-reports for psychosocial work conditions were obtained in a sample of sawmill workers using the demand/control and effort/reward imbalance models. The relative predictive validity of task-level control was compared with effort/reward imbalance. As well, the predictive validity of a model developed by combining task-level control with effort/reward imbalance was determined. Logistic regression was utilized for all models.

Results: The demand/control and effort/reward imbalance models independently predicted poor self-reported health status. The effort-reward imbalance model predicted the presence of a chronic disease while the demand/control model did not. A model combining effort-reward imbalance and task-level control was a better predictor of self-reported health status and any chronic condition than either model alone. Effort reward imbalance modeled with intrinsic effort had marginally better predictive validity than when modeled with extrinsic effort only.
\end{abstract}

Conclusions: Future work should explore the combined effects of these two models of psychosocial stress at work on health more thoroughly.

\section{Background}

A strong body of evidence indicates that exposure to adverse psychosocial work conditions is a major hazard for the health of workers in modern economies. Much of this evidence, accumulated over the past two decades, is based on the demand/control model [1] in which task-level work conditions characterized by low control and high demand have been shown to predict high rates of cardio- vascular disease as well as high rates of sickness absence $[2,3]$.

One of the criticisms of this model is its reliance on "objective" measures of the work environment only [4]. According to many critics, workers will respond differently to the same constellation of control and demand conditions leading to varied biological outcomes so that a measure of individual worker differences, specifically in 
coping style, must therefore be included in any job strain model.

In the early 1990s, the effort-reward imbalance model was developed [5]. This model postulates that jobs characterized by a perceived imbalance between high effort and low rewards are stressful and will lead to negative health outcomes, particularly in persons with limited coping abilities. This model is meant to tap the attribute of an individual's "need for control"; a personality characteristic related to flexibility in coping. According to the model, a person with high need for control will respond in an inflexible way to work situations of high effort and low reward; and will therefore be more stressed and disease prone than a person in the same situation who has less need for control.

Using well designed epidemiological studies both models have succesfully predicted "hard" disease outcomes (particularly CHD) $[6,7]$. In the first comparative study with both models, Bosma has demonstrated independent predictive effects for new coronary heart disease of a component of the demand/control model (low control) as well as effort/reward imbalance in a cohort of English whitecollar workers [8].

The models overlap to some extent as "extrinsic demands" in the effort/reward imbalance model is similar to "psychological and physical demands" in the demand/control model. However, the models also differ. The effort/reward imbalance model includes a measure of coping ability (need for control) which has no counterpart in the demand/control model. On the other hand, effort/reward imbalance excludes any measurement of task-level control. This is important, for as Bosma notes, "recent publications increasingly underscore the special importance of low job control for a range of health outcomes, including cardiovascular disease and sickness absence" [[8] p68].

The purpose of this investigation is to compare the predictive validity of the demand/control and effort reward/imbalance models for self-reported health status and the selfreported presence of any chronic disease condition in a sample of former and current sawmill manufacturing workers. As well, because task-level control is the only element which is absent from the effort/reward imbalance model, and because this variable has been consistently predictive of a range of health outcomes for the past 2 decades, the predictive validity of the effort/reward imbalance model in combination with task-level control is also tested.

\section{Methods}

This investigation is based on a sample of 3,000 male sawmill workers drawn randomly from a cohort that was orig- inally gathered to study the impact of chlorophenol antisapstain chemicals on British Columbia (BC) sawmill workers $[9,10]$.

\section{Selection of sawmills and workers for the original study}

Fourteen medium to large sized sawmills, located mainly in Southwest BC, participated in the original cohort study. Study sawmills were selected on the basis of a long-term history of chlorophenol use and availability of intact personnel records. A total of 26,221 workers were enrolled in the cohort, representing approximately 20 percent of all BC sawmill workers. (This increased to approximately 29,000 as workers hired in study mills between 1986 and 1996 were added to the cohort.) To be eligible, a worker had to be employed at a study mill for at least one year between January 1, 1950 and December 31, 1996.

The investigation of this sample of 3,000 workers was originally designed to study the impact of a recession and major restructuring of sawmills which began in 1980. Accordingly, the year 1979 was chosen as the pre-recession/ restructuring "baseline" year. All workers enrolled in the cohort during 1979 were included in this baseline sub-cohort. A sample of 3,000 workers was randomly selected from the 9,806 workers working in a study sawmill in 1979 .

\section{Locating interviewees}

In order to locate interviewees the 1979 sub-cohort was linked to the British Columbia Linked Health Database (BCLHDB). This database includes provincial health ministry files on physician services, hospital discharges, drug prescriptions for the elderly, long term care services, deaths, and births for the years 1985 through to 1996 . These data are useful for finding individuals because they include patient postal codes at time of contact with a physician. Ethical approval limited our access to the first 3digits of the 6-digit postal codes. This allowed us to identify the community where cohort members lived, so that we could then find them through public information sources.

The 9,806 workers employed at a study mill in 1979 were linked probabilistically to the BCLHDB. Linkage efficiency was $94.7 \%$ so that 3 -digit postal codes were obtained for 9,282 workers; including 2,920 (97.3\%) of the 3000 randomly sampled workers. Searches of union pension plans, electronic telephone databases, and telephone books (by hand) were undertaken to obtain full addresses for the 3,000 workers. For the unlinked workers in the sample, address searches were undertaken using names only. 
Table I: Interview status of 3,000 randomly sampled workers

\begin{tabular}{lll}
\hline Interview Status & Number & Percent \\
\hline Long questionnaire & 1885 & $\mathbf{6 2 . 9}$ \\
Short questionnaire & $\mathbf{2 7 0}$ & $\mathbf{9 . 1}$ \\
Questionnaire Respondents sub-total & 2155 & 72.0 \\
Refusals & 126 & 4.2 \\
Deceased & 18 & $\mathbf{0 . 6}$ \\
Needs translator & $\mathbf{8}$ & $\mathbf{0 . 3}$ \\
Not located & $\mathbf{6 9 3}$ & $\mathbf{2 2 . 8}$ \\
Total & $\underline{3000}$ & $\underline{100.0}$ \\
\hline
\end{tabular}

\section{Administering the interviews}

Face-to-face interviews were conducted between November 1997 and March 1999. Subjects living in remote regions of the province were interviewed by telephone. A short version of the questionnaire (requiring about 20 minutes compared to one hour) was administered by telephone when a respondent was only willing to conduct a brief interview or when proxy interviews were conducted for deceased or incapacitated interviewees. However, because work-related variables were incompletely determined with the short version of the questionnaire, only the long version of the questionnaire was used in this investigation.

\section{The instrument}

The instrument was developed after a thorough review of the literature on technological change, restructuring, unemployment, and health and work. Two focus groups were conducted with experienced sawmill workers to finalize the questionnaire; it was then pilot tested on 29 retired sawmill workers.

Socio-demographic information and health behaviours (smoking and alcohol consumption) were measured. Income was measured for the year preceding interview as was the number of dependants supported by each subject. Income per dependant over the year prior to interview was categorized into quartiles. Education status was categorized as completed elementary, secondary, apprentice training, community college, and university.

Because of downsizing/restructuring over the follow-up period, many workers moved out of the sawmill sector and in and out of employment. Variables measuring nonpsychosocial work conditions, which may confound associations between current psychosocial work conditions and self-reported health outcomes, such as history of unemployment, sector of employment and occupational category at time of interview were developed as follows. Data on current work sector was obtained and dichotomized (currently employed in the sawmill sector vs currently em- ployed outside the sawmill sector). Data on unemployment history was also obtained and categorized as follows: no unemployment, 1 episode less than 1 year in duration, 1 episode greater than 1 year in duration, 2 episodes or more less than 1 year in duration, 2 episodes of more greater than 1 year in length.

All job titles obtained in the interviews were re-coded using the Canadian Standard Occupational Classification [12] and then translated into the Pineo16 Occupational Status Scale [13]. This 16 category scale was collapsed into 4 basic categories; professional/managerial, trades, semiskilled, and unskilled to measure current occupational category.

Task-level work characteristics were measured using a shortened version [10] of Karasek's demand/control instrument $[14,15]$. Scores for control and psychological demand were divided into high and low categories at the median. Jobs which were high in psychological demand and low in control were categorized as high strain.

Esteem reward, status control, extrinsic effort, and need for control were measured in the "full" effort/reward imbalance model [5]. The two effort scales were constructed by summing across questions and dichotomizing the scale score with zero for the two bottom tertiles and one for the highest tertile representing high extrinsic effort and high need for control. Two reward scales were constructed by summing across questions and dichotomizing the scale score with zero for the two top tertiles and one for the bottom tertile representing low esteem reward and low status control. This "full" model was thus based on four variables, intrinsic demand, extrinsic demand, esteem reward, and status control. These four dichotomous variables were used to create the effort/reward imbalance indicators consisting of three categories: $1=$ neither high effort nor low reward; $2=$ either high effort or low reward; and 3=both high effort and low reward used in the "full" effort/reward imbalance model[ $[5,8,16]$. 
Table 2: Age and labour force status of long questionnaire respondents at time of interview

\begin{tabular}{lll}
\hline Current Status & Number & Percent \\
\hline Age Status & & \\
65 Years or Over & 464 & 24.6 \\
64 Years or Less & 1421 & 75.4 \\
Total & $\mathbf{1 8 8 5}$ & 100.0 \\
Labour force Status & & \\
Sawmill Sector & 600 & 42.3 \\
Non-Sawmill Sector & 570 & 40.1 \\
Early Retired & 131 & 9.2 \\
Unemployed & 69 & 4.8 \\
Disabled & 40 & 2.8 \\
Other & 11 & 0.8 \\
Total & $\mathbf{1 8 8 5}$ & $\mathbf{1 0 0}$ \\
\hline
\end{tabular}

A "partial" effort/reward imbalance model was also developed by eliminating one of the four components, intrinsic effort, from the full model. This "partial" model was thus based on three variables of the variables (extrinsic demand, esteem reward, and status control) utilized in the full model. Because the demand/control model contains no information on the personal characteristics of the worker, using a partial effort/reward imbalance model, i.e., with the psychological measure (intrinsic demand) removed, allows for a comparison of the "objective" elements of both models.

The combined effect of task-level control and the effort-reward imbalance models (full and partial) was tested by categorizing effort/reward imbalance into three categories (none, medium, and high) and task-level control into the two categories high and low. In this way workers were categorized into, at one extreme, a reference category of no effort/reward imbalance and high task-level control and, at the other extreme, a category of high effort/reward imbalance and low task-level control with 4 categories representing the possible combinations of effort/reward imbalance and task-level control between the two extremes.

Two outcome variables were used in this investigation; self-reported health status, and the presence of one self-reported chronic condition. Self-reported health was reported on a 5 point scale for current job and dichotomized into "good" (good or excellent) and "poor" (fair, poor, bad) health status for use in logistic regression analysis. Self-reported health is dichotomized, with a cut point between good and fair in most studies of work stress of this type [17]. And, any worker who reported one or more of the following conditions at time of interview was considered to have a chronic disease: asthma, back problems (excluding arthritis), chronic bronchitis or emphysema, diabetes, CHD, hearing loss; or any other non-specified chronic condition.

\section{Analyses}

Logistic regression was used to determine the association between self-reported health status or self-reported chronic disease status and with various exposure variables. In the first model, the association between both outcome variables and sociodemographic variables was examined (Table 3). In the second model, sociodemographic variables and current smoking status were included as adjustment variables in a model examining the association between the outcome variables and three non-psychosocial work variables, current sector, current occupational category, and unemployment history (Table 4).

In the third model, after controlling for socio-demographic variables, smoking, and non-psychosocial work conditions, associations with demand/control and effort/ reward imbalance (full and partial) were tested (Table 5). In the fourth model associations were tested after combining effort/reward imbalance (full and partial) with tasklevel control (Table 6, 7).

\section{Results}

Table 1 shows that the overall response rate was 72 percent, the refusal rate was 4.2 percent, and $19 \%$ of respondents were not located. The proportion of workers not located was highest in isolated "mill towns" with a younger more transient workforce than at other sawmills in the cohort. Refusal rates did not vary by age category but the "not found" rate was higher in younger age groups and workers with lowest duration of work in a study sawmill.

Some respondents did not speak English well enough for the interview. Cantonese and Punjabi speaking translators were hired to administer interviews to subjects who spoke 
Table 3: Age adjusted associations of socio-demographic and smoking factors with poor self-reported health status (SRHS) and selfreported chronic condition (Chronic).

\begin{tabular}{|c|c|c|}
\hline Variable & Chronic & SRHS \\
\hline \multicolumn{3}{|l|}{ Age } \\
\hline $35-39$ & 1.00 & 1.00 \\
\hline $40-44$ & 0.99 & $1.69 *$ \\
\hline $45-49$ & 1.37 & $1.77^{*}$ \\
\hline $50-54$ & $1.88^{* *}$ & $1.72 *$ \\
\hline $55-59$ & $2.32 * *$ & $2.06 * *$ \\
\hline $60-64$ & $4.25^{* *}$ & $2.50 * *$ \\
\hline \multicolumn{3}{|l|}{ Birthplace } \\
\hline Canada & 1.00 & 1.00 \\
\hline Outside Canada & $0.66 * *$ & 1.32 \\
\hline \multicolumn{3}{|l|}{ Education } \\
\hline Elementary & 1.00 & 1.00 \\
\hline Secondary & 0.99 & 0.75 \\
\hline Apprentice & 1.34 & 0.76 \\
\hline Community College & 1.05 & 0.75 \\
\hline University & 0.71 & $0.60 *$ \\
\hline \multicolumn{3}{|l|}{ Marital status } \\
\hline Married & 1.00 & 1.00 \\
\hline Unmarried & 1.08 & 1.05 \\
\hline \multicolumn{3}{|l|}{ Income/dependant } \\
\hline$<\$ 12,999$ & 1.00 & 1.00 \\
\hline$\$ 13,000-18,749$ & 1.31 & 0.87 \\
\hline$\$ 18,750-28,229$ & 1.34 & 0.75 \\
\hline$>\$ 28,00$ & 1.20 & $0.65^{*}$ \\
\hline \multicolumn{3}{|c|}{ Current Smoking status } \\
\hline No & 1.00 & 1.00 \\
\hline Yes & 1.11 & $1.55^{* *}$ \\
\hline
\end{tabular}

$*_{p}=0.05-0.01 ; *_{p}=<0.01$

Table 4: Socio-demographic and smoking adjusted associations of non-psychosocial work condition variables with poor self-reported health status and self-reported chronic condition.

\begin{tabular}{lll}
\hline Variable & Chronic & SRHS \\
\hline Sector & 1.00 & 1.00 \\
Non-Sawmill & 1.09 & $1.57^{* *}$ \\
Sawmill & & 1.00 \\
Current Occupational Category & 1.00 & 1.08 \\
Manager & 1.30 & 1.26 \\
Trades & 0.89 & 1.00 \\
Unskilled & 1.00 & 1.12 \\
Unemployment History & 1.19 & 0.88 \\
I episode< I year & 1.01 & 1.22 \\
I episode $>$ I year & 1.21 & \\
2 episodes or more $<1$ year & & \\
2 episodes or more $>1$ year & & \\
\hline
\end{tabular}

$*_{p}=0.05-0.01 ; *_{p}=<0.01$

these languages. Eight people, with other languages required translators but due to expense and logistics these interviews were not conducted.
Table 2 shows that $1,421(75.4 \%)$ of the 1,885 long questionnaire respondents were aged 64 or less. Of these, 1170 $(82.3 \%)$ were employed at time of interview, 131 (9.2\%) 
Table 5: Associations between the demand/control and effort/reward imbalance models (full and partial) and poorself-reported health status and any chronic condition.

\begin{tabular}{|c|c|c|}
\hline & Self-reported Health Status & Any Chronic condition \\
\hline \multicolumn{3}{|l|}{ Control } \\
\hline High & 1.00 & 1.00 \\
\hline Low & $1.60 * *(1.12-2.28)$ & $1.09(0.81-1.45)$ \\
\hline \multicolumn{3}{|c|}{ Psychological demand } \\
\hline Low & 1.00 & 1.00 \\
\hline High & $1.65^{* *}(1.21-2.26)$ & $1.13(0.86-1.48)$ \\
\hline \multicolumn{3}{|c|}{ Physical demand } \\
\hline Low & 1.00 & 1.00 \\
\hline High & $1.01(0.68-1.50)$ & $1.25(0.86-1.81)$ \\
\hline \multicolumn{3}{|l|}{ Job Strain } \\
\hline Low & 1.00 & 1.00 \\
\hline High & $2.07 *(1.18-3.66)$ & $1.31(0.77-2.22)$ \\
\hline \multicolumn{3}{|c|}{ Partial Effort/reward Imbalance Model } \\
\hline None & $1.00 * *$ & $1.00 *$ \\
\hline Medium & $1.60 *(1.06-2.45)$ & $1.12(0.0 .84-1.44)$ \\
\hline High & $3.13^{* *}(1.96-4.85)$ & $1.59 * *(1.12-2.24)$ \\
\hline \multicolumn{3}{|c|}{ Full Effort/reward Imbalance Model } \\
\hline None & $1.00 * *$ & $1.00 * *$ \\
\hline Medium & $1.87^{* *}(1.26-3.34)$ & $1.22(0.91-1.85)$ \\
\hline High & $3.35^{* *}(2.10-5.61)$ & $1.70 * *(1.17-2.37)$ \\
\hline
\end{tabular}

Numbers in parentheses are $95 \%$ confidence intervals. ${ }^{*} p=0.05-0.01 ; * * p=0.01-0.0001$

Table 6: Associations between effort/reward imbalance (full and partial) model combined with task-level control and poor self-reported health status

\begin{tabular}{|c|c|c|}
\hline & Full Model & Partial Model \\
\hline NO Imbalance with HIGH control & $I^{* *}$ & I** \\
\hline NO Imbalance with LOW control & $0.75(0.37-1.48)$ & $0.7 \mid(0.4|-| .25)$ \\
\hline MEDIUM Imbalance with HIGH control & $1.40(0.77-2.69)$ & $1.16(0.66-2.02)$ \\
\hline MEDIUM Imbalance with LOW control & $1.74(0.97-3.10$ & $1.54(1.07-2.55)$ \\
\hline HIGH Imbalance with HIGH control & $2.20 * *(1.25-3.99)$ & $2.09 * *(1.25-3.50)$ \\
\hline HIGH Imbalance with LOW control & $3.50 * *(2.04-6.08$ & $3.23^{* *}(2.01-5.18)$ \\
\hline
\end{tabular}

Numbers in parentheses are $95 \%$ confidence intervals. ${ }^{*} \mathrm{p}=0.05-0.01 ; *^{*} \mathrm{p}<0.01$

were retired, $69(4.9 \%)$ were unemployed, $40(2.8 \%)$ were disabled, and $11(0.8 \%)$ were in at home looking after children, performing voluntary work, or attending educational institutions at time of interview. Of the 1170 workers employed at time of interview, 600 (51.3\%) were employed in a sawmill and $570(48.7 \%)$ were employed outside the sawmill sector. The analyses described in this paper were based on the 1170 respondents who answered the long questionnaire and who were aged 64 or under and employed at time of interview.

Table 3 presents age-adjusted associations between self-reported health status and chronic conditions and sociodemographic variables, smoking, and self-reported health status in 1979. The presence of a chronic condition was associated in a step-wise gradient with increasing age. For the remaining variables, no statistically significant associations were observed with either outcome variable.

Self-reported health status declined step-wise with increasing age. Increasing education was positively associated, in gradient fashion, with increasing self-reported health status. This was statistically significant for university education $(\mathrm{OR}=0.60)$. Current smoking was associated with worse self-reported health status $(\mathrm{OR}=1.55)$.

Table 4 shows that, after adjustment, none of the non-psychosocial work variables demonstrated a statistically significant relationship with the presence of a chronic condition. In the case of self-reported health status, cur- 
Table 7: Associations between effort/reward imbalance (full and partial) model combined with task-level control and poor self-report of any chronic condition

\begin{tabular}{ccc}
\hline & Full Model & Partial Model \\
\cline { 2 - 3 } NO Imbalance with HIGH control & $1 *$ & 1 \\
NO Imbalance with LOW control & $1.04 * 0.63-1.72)$ & $0.97(0.67-1.58)$ \\
MEDIUM Imbalance with HIGH control & $1.13(0.70-1.83)$ & $1.0(0.65-1.56)$ \\
MEDIUM Imbalance with LOW control & $1.38(0.83-2.27)$ & $1.22(0.77-1.93)$ \\
HIGH Imbalance with HIGH control & $1.57(0.95-2.49)$ & $1.38(0.86-2.20)$ \\
HIGH Imbalance with LOW control & $1.98 * *(1.23-3.18)$ & $1.80^{*}(1.14-1.80)$
\end{tabular}

Numbers in parentheses are $95 \%$ confidence intervals. ${ }^{*} p=0.05-0.01 ; * * p<0.01$

rent employment in the sawmill sector was associated with greater odds (statistically significant) for reporting poor health $(\mathrm{OR}=1.57)$ and decreasing occupational status was associated with a (non-significant) gradient for worse self-reported health.

Table 5 shows that low control $(\mathrm{OR}=1.60$; $\mathrm{CI}=1.12-$ 2.28 ) and high psychological demand $(\mathrm{OR}=1.65 ; \mathrm{CI}=$ 1.21-2.26) predicted poor self-reported health. Effort/reward imbalance and job strain both predicted poor health status. The risk of reporting poor health status for subjects with high job strain was approximately twice as high as those with low strain $(\mathrm{OR}=2.07 ; \mathrm{CI}=1.18-3.66)$. And, for both the full and partial effort/reward imbalance models the risk of reporting poor health status for subjects with both high effort and low reward was approximately 3 times higher than those with low effort and high reward (full model; $\mathrm{OR}=3.35 ; \mathrm{CI}=2.10-5.51$ ) and (partial mod$\mathrm{el} ; \mathrm{OR}=3.13 ; \mathrm{CI}=1.96-4.85)$.

Effort/reward imbalance (full and partial models) was the only variable which predicted the presence of a chronic condition. The risk of reporting a chronic condition for subjects with both high effort and low reward was 59 percent greater than those with low effort and high reward in the case of the partial model and 70 percent greater with the full model. The risk of reporting a chronic condition for subjects with high job strain was approximately $30 \%$ greater than those with low strain jobs.

For self-reported health status, combining effort/reward imbalance with task-level control produced odds ratios which increased in a regular gradient moving from the reference category (no effort/reward imbalance with high task-level control) to the "worst" category (effort/reward imbalance with low task-level control) (Table 6). For the full effort/reward imbalance model, the odds ratio for this latter category was $3.50(\mathrm{CI}=2.04-6.08)$. For the partial model the odds ratio for this latter category was $3.23(\mathrm{CI}=$ 2.01-5.18). In the case of chronic conditions, for the full effort/reward imbalance model, the odds ratio for the worst" category (effort/reward imbalance with low tasklevel control) was $1.98(\mathrm{CI}=1.23-3.18)$ and for the partial model the odds ratio was $1.80(\mathrm{CI}=1.14-1.80)$ (Table 7$)$.

Finally, in the case of self-reported health status, the Model Chi Square for the full effort/reward imbalance model combined with task-level control was 71.95 compared to 65.1 for effort/reward imbalance alone. Results were similar in size and trend for the partial effort/reward imbalance model combined with task-level control. In the case of "any chronic condition" the Model Chi Square for the full effort/reward imbalance model combined with tasklevel control was 46.67 compared to 44.97 for effort/reward imbalance alone. Results were similar in size and trend for the partial effort/reward imbalance model combined with task-level control

\section{Discussion}

In this investigation, effort/reward imbalance and job strain independently predicted self-reported health status and both the full and partial effort/reward imbalance models predicted the presence of a chronic condition. As well, both the full and partial effort/reward imbalance models in conjunction with task-level control predicted self-reported health status and the presence of a chronic condition.

The odds ratio for self-reported health status with the combination high effort/reward imbalance (full model) and low task-level control was 3.50 and the odds ratio in the case of the partial effort/reward imbalance model in combination with low task-level control was 3.23. These odds ratios were slightly higher than those obtained using the effort/reward imbalance model alone and approximately 50 percent greater than odds rations obtained using the demand control model (i.e., the job strain variable) alone. Similarl results were obtained for the outcome "any chronic condition".

Is the combined model (effort/reward imbalance with task-level control) a better predictor of the two outcome 
variables than effort/reward imbalance or task-level control alone? As noted, odds ratios were slightly higher for the combined models compared to effort/reward imbalance and task-level control on their own. The full effort/ reward imbalance task-level control model explained $11.7 \%$ and $41.1 \%$ more variance in self-reported health status than the effort/reward imbalance model and tasklevel control alone. Results for the partial effort/reward imbalance task-level control model were similar. And, similar, but less pronounced trends were observed for the outcome "any chronic condition". These results both confirm those obtained from the Whitehall study $[8,18]$ and extends it.

The predictive ability of the full compared to the partial (i.e., without intrinsic effort) effort/reward imbalance model was only marginally greater for both health outcomes. While intrinsic effort is a major theoretical component of the effort/reward imbalance model, its did not, in this study, contribute markedly to enhanced predictive ability for the model. The use of the partial effort/reward imbalance model should be further explored with other data, with "hard" outcomes, to determine empirically whether or not intrinsic effort adds substantially to the predictive ability of the model.

Major strengths of this study are that 1) both effort/reward imbalance and demand/control measures were obtained for each individual, 2 ) the sample was large (1,170 workers), 3) the workers, were at the time of the survey, employed across many sectors including manufacturing and the service, transportation, and construction sectors, and 4 ) the occupational status of workers ranged from unskilled to skilled professionals. Thus, in spite of the original sampling frame (which meant that all those sampled had been employed in the sawmill industry approximately 20 years prior to the survey), the sample represented a fairly heterogeneous group of middle-aged, male, former resource manufacturing workers.

The most serious, limitation of this study arises because both explanatory and outcome variables are based on selfreports at time of interview. It is possible that "soft" dependent variables such as self-reported health status may derive from the same conception of self as explanatory variables like psychosocial work conditions. In this case, there is a problem of common methods' variance in which the independent and dependent variables are hardly distinguishable [19] resulting in the possibility of contamination between measures [20].

Bias arising from common methods' variance may be a greater problem for the full effort/reward imbalance model relative to both the partial effort/reward balance model and the demand/control model. The intrinsic effort meas- ure included in the full effort-reward imbalance model is essentially a measure of coping ability which because of its subjective nature may be more vulnerable to common methods' variance than the other variables in the effort/reward and demand/control models. Use of the partial effort/reward imbalance model may mitigate any common method's variance related to the intrinsic effort variable.

As well, some researchers have argued that any associations observed between self-reports of psychosocial work conditions and health outcomes may be confounded by the subjective "state" or personality of the worker [2123]. According to this perspective, the major factor responsible for this confounding is "negative affectivity" and that the impact of this confounding is so great that self-reports of job work conditions are essentially a measure of negative affectivity [21].

However Bosma has demonstrated, using the demand/ control model and data from the Whitehall study, "an absence of consistently stronger effects of job control in participants with reported negative personal characteristics [which] also indicates that a neurotic tendency to complain cannot explain the job control-CHD association" [[24] p406]. These and other recent findings have demonstrated that it may not be useful to measure and control for negative affectivity in studies using self-reports of psychosocial work conditions [25].

\section{Conclusions}

In summary, task-level control and effort-reward imbalance were independently associated with self-reported health status and effort reward imbalance was associated with self-reported presence of any chronic condition. Modeled in combination, effort/reward imbalance and task-level control was more predictive for both outcomes than the effort/reward imbalance and demand/control models alone. The predictive power of the full effort/reward imbalance model was only marginally greater than for the partial model using both health outcome measures. Future work should explore the combined effects of these two models of psychosocial stress at work on health more thoroughly.

\section{Competing interests}

None declared.

\section{Authors' contribution}

AO conducted the research, organized data gathering, and wrote the paper. SK carried out data cleaning tasks. PD provided guidance in study design. CM provided writing help and conceptual design. $\mathrm{CH}$ provided conceptual help. 


\section{Note}

${ }^{1}$ In this study effort and reward variables were constructed from questionnaire items which were similar to but were not the same as items used by Johannes Siegrist in his effort/reward imbalance instrument.

\section{Acknowledgments}

I'd like to thank Dr. Johannes Siegrist and Dr. Micky Kerr for their help in reviewing this paper. Acknowledgments to the Canadian Institute of Health Research and Micheal Smith Foundation for Health Research for Dr. Ostry's salary support. As well, acknowledgments to the Canadian Population Health Initiative, the Institute of Work and Health, the Canadian Institute of Advanced Research, the Center for Health Services and Policy Research, and Forest Renewal British Columbia for their financial and intellectual support for this project.

\section{References}

I. Karasek R Job demands, job decision latitude, and mental strain: Implication for job redesign. Admin Sci Quart 1979, 24:285-308

2. Landsbergis PA, Schnall P, Schwartz J, Warren K and Pickering TG Job strain, hypertension, and cardiovascular disease: Recommendations for further research. In Organisational risk factors for job stress (Edited by: Sauter SL, Murphy LR) Washington: American Psychological Association 1995, 97-II2

3. North F, Syme L, Feeney A, Shipley M and Marmot M Psychosocial work environment and sickness absence among British civil servants: The Whitehall II study. Am J Public Health 1996, 86:332340

4. Frese $M$ and Zapf $D$ Methodological issues in the study of work stress: Objective vs subjective measurement of work stress and the question of longitudinal studies. In: Causes, coping, and consequences of stress at work (Edited by: Cooper C, Payne R) New York:John Wiley \& Sons Ltd 1988, 375-4I I

5. Siegrist J, Peter R, Junge A, Cremer P and Seidel D Low status control, high effort at work and ischemic heart disease: Prospective evidence from blue-collar men. Soc Sci \& Med 1990, 3 I (I0): I I 27-I I 34

6. Karasek R and Theorell T Healthy work. New York: Basic Books 1990,

7. Siegrist J Adverse health effect of high-effort/low-reward conditions. Occup Health 1996, I:27-4 I

8. Bosma H, Peter R, Siegrist J and Marmot M Two alternative job stress models and the risk of coronary heart disease. Am J Public Health I998, 88(I):68-74

9. Hertzman C, Teschke K, Ostry A, Dimich-Ward H, Kelly S and Spinelli J Mortality and cancer incidence in sawmill workers exposed to chlorophenols. Am J Public Health 1996, 87:71-79

10. Ostry A, Marion SA, Green L, Demers PA, Hershler R and Kelly S Technological change in relation to changes in psychosocial conditions of work in BC sawmills (1950-1996). Scand J Work Environ Health 2000, 26(3):273-277

1I. Ostry A, Marion SA, Green L, Demers PA, Hershler R and Kelly S Comparison of expert-rater methods for assessing psychosocial job strain. Scand J Work Environ Health 200I, 27(I): I-6

12. Statistics Canada. Standard occupational classifications, Ottawa 1980 ,

13. Pineo P Revisions of the Pinio-Porter-McRoberts socioeconomic classification of occupations for the 1981 census, QSEP Research Report No. 125, Program for Quantitative Studies in Economics and Population, McMaster University, Hamilton, Ontario February 1985

14. Karasek R, Gordon G, Pietrokovsky C, Frese M, Pieper C and Schwartz J Job content instrument: questionnaire and users' guide. Lowell(MA): University of Massachusetts 1985,

15. Johnson J, Hall $E$ and Theorell $T$ Combined effects of job strain and social isolation on cardiovascular disease morbidity and mortality in a random sample of the Swedish male working population. Scand J Work Environ Health 1989, I5:27I-279

16. Stansfeld S, Bosma H. Hemingway $\mathrm{H}$ and Marmot M Psychosocial work characteristics and social support as predictors of SF36 health functioning: the Whitehall II study Psychoom Med $1998,60: 247-255$
17. Kelly S Self-reported Health: Stitching together a picture from the fabric of life. P.h.D. Thesis, University of British Columbia. 2003,

18. de Jonge J, Bosma H, Peter R and Siegrist J Job strain, effort-reward imbalance and employee well-being: a large scale cross-sectional study. Soc Sci \& Med 2000, 50: I 3 | 7 - 1327

19. Kasl S Measuring job stressors, and studying the health impact of the work environment: an epidemiologic commentary. J Occup Health Psychol 1998, 3(4):390-40 I

20. Kristensen $T$ Job stress and cardiovascular disease: a theoretic critical review. J Occup Health Psychol 1996, I(3):246-260

21. Chen PY, Spector PE and Jex SM Effects of manipulated job stressors and job attitude on perceived job conditions: $\mathrm{A}$ simulation. In: Organizational risk factors for job stress (Edited by: Sauter SL, Murphy LR) Washington D.C.: American Psychological Association 1995,

22. Spector P, Dwyer D and Jex S Relation of job stressors to affective, health, and performance outcomes: a comparison of multiple data sources. J Appl Psychol 1988, 73:11-19

23. Spector $P$ and Jex $S$ Relations of job characteristics from multiple data sources with employee affect, absence, turnover intentions, and health. J Appl Psychol I99I, 76:46-53

24. Bosma $\mathrm{H}$, Stansfeld $\mathrm{S}$ and Marmot $\mathrm{M}$ Job control, personal characteristics, and heart disease. J Occup Health Psychol 1998, 3(4):402-409

25. Spector $P$, Zapf $D$, Chen $P$ and Frese $M$ Why negative affectivity should not be controlled in job stress research: don't throw out the baby with the bath water. J Org Behaviour 2000, 21:79-95

\section{Pre-publication history}

The pre-publication history for this paper can be accessed here:

\section{http://www.biomedcentral.com/1471-2458/3/10/prepub}

Publish with Biomed Central and every scientist can read your work free of charge

"BioMed Central will be the most significant development for disseminating the results of biomedical research in our lifetime. "

Sir Paul Nurse, Cancer Research UK

Your research papers will be:

- available free of charge to the entire biomedical community

- peer reviewed and published immediately upon acceptance

- cited in PubMed and archived on PubMed Central

- yours - you keep the copyright 